\title{
Magnetically controlled circumstellar plasma in the helium-weak stars ${ }^{\star}$
}

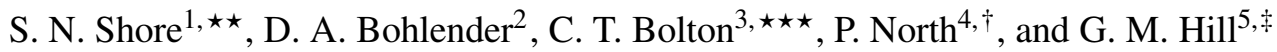 \\ 1 Department of Physics "Enrico Fermi”, University of Pisa, via Buonarroti 2, Pisa 56127, Italy \\ ${ }^{2}$ Herzberg Institute of Astrophysics, National Research Council of Canada, 5071 West Saanich Road, Victoria, BC, \\ Canada V9E 2E7 \\ e-mail: david.bohlender@nrc-cnrc.gc.ca \\ 3 David Dunlap Observatory, University of Toronto, PO Box 360, Richmond Hill, Ontario L4C 4Y6 Canada \\ e-mail: bolton@astro.utoronto.ca \\ ${ }^{4}$ Laboratoire d'Astrophysique, École Polytechnique Fédérale de Lausanne, Observatoire, 1290 Chavannes-des-Bois, \\ Switzerland \\ e-mail: Pierre.North@obs. unige.ch \\ 5 W. M. Keck Observatory, 65-1120 Mamalahoa Hwy, Kamuela, HI 96743, USA \\ e-mail: ghill@keck.hawaii.edu
}

Received 2 November 2003 / Accepted 30 March 2004

\begin{abstract}
We report the discovery of variable C IV line profiles in the magnetic helium-weak stars HD 142301, HD 142990, and HD 144334, all of which are members of the Sco-Cen association, and the hot helium-weak star HD 175362. These stars display behavior that is similar to HD 5737 and HD 79158, two helium-weak stars for which magnetically confined plasma has previously been reported. The present study thus extends the C IV survey to all of the stars for which strong radio emission has been detected. No C IV absorption has been detected for two other Sco-Cen chemically peculiar stars, HD 144844 and 146001, nor has it been detected from archival spectra of HD 28843, HD 49333, or HD 124224. The reported detections are the first helium-weak stars that do not belong to the $s n$ subclass that have been found to display such UV line profile variations. It appears that the restriction of this phenomenon to the extreme helium-weak subgroup was a selection effect and that the occurrence of magnetically trapped corotating plasma is more widespread among the upper main sequence magnetic spectrum variables. We show model calculations for the C IV variability that clarify the plasmaspheric structure. Supporting $\mathrm{H} \alpha$ observations show no evidence of line profile variability for HD 142301 and HD 144334, while HD 142990 appears to have extremely weak emission at certain phases, and the profile of HD 124224 shows some changing asymmetry.
\end{abstract}

Key words. stars: chemically peculiar - stars: magnetic fields - stars: atmospheres - circumstellar matter

\section{Introduction}

The upper main sequence helium-peculiar stars are the hottest of the magnetic chemically peculiar (CP) stars. They show a wide range of interesting phenomena, unique among single, non-accreting stars. In addition to spectrum and photometric variations, frequently encountered among the other

Send offprint requests to: S. N. Shore, e-mail: shore@df.unipi .it

* Based on observations collected at the European Southern Observatory, La Silla, Chile (ESO programme No. 53.7-0009).

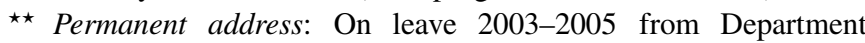
of Physics and Astronomy, Indiana University South Bend, 1700 Mishawaka Avenue, South Bend, IN 46634-7111; Guest observer, International Ultraviolet Observatory.

$\star \star \star$ Guest observer, International Ultraviolet Observatory.

$\dagger$ Guest observer, European Southern Observatory.

Visiting Astronomer, Canada-France-Hawaii Telescope, which is operated by the National Research Council of Canada, the Centre National de la Recherche Scientifique of France, and the University of Hawaii. magnetic $\mathrm{CP}$ stars, the hotter stars also show signatures of complex circumstellar environments including plasmaspheres, magnetically collimated outflows, and radio emission. Some of this behavior can be understood in the context of the model developed for the helium-strong stars in which a stellar wind is diverted from purely radial outflow by the strong surface magnetic field (Shore 1987). The field regulates the density and velocity of the circumstellar plasma, producing jet-like flows above the magnetic pole (where the field lines are open) and magnetospheric trapping at the magnetic equator.

For the helium-strong stars, all of which have spectral types earlier than B2, the behavior is consistent with the expected mass loss rates for main sequence stars driven by line mediated radiation pressure. The mass loss is also apparently necessary for explaining the segregation of helium over the stellar surface (Vauclair 1975; Shore 1987; Vauclair et al. 1991) and possibly in the winds (Groote \& Hunger 1999). One O-star, $\theta^{1}$ Ori, has also been found to display a corotating plasmasphere (see Donati et al. 2002). For the helium-weak stars, however, 
the model predicts that the plasmasphere should not be strongly populated because the stellar wind is very feeble, too weak to support the helium overabundances seen in the hotter CP stars.

Optical and ultraviolet studies of the helium-strong stars (e.g., Groote \& Hunger 1976, 1982; Bolton 1984; Bolton et al. 1986; Shore \& Brown 1990; Leone \& Umana 1993; Bolton \& Short 1994; Wade et al. 1997; Bolton et al. 1998) show that these stars display all the signatures of magnetically controlled circumstellar plasma - NLTE-enhanced resonance lines of the ions C IV, Al III, and Si IV, variable $\mathrm{H} \alpha$ line emission, and enhanced shell cores in the higher Balmer series members whose strengths depend on the magnetic phase. The period variations are well modeled within the oblique rotator picture (Shore \& Brown 1990; see also Shore 1999). For cooler B stars, a centrifugally driven wind is a possible source for circumstellar plasma (Mestel 1968). The helium-weak stars do not possess sufficiently high ultraviolet fluxes to radiatively drive a stellar wind. The most rapidly rotating magnetic stars might then be expected to show more circumstellar gas, and there should be a strong dependence of the density of the magnetospheric gas on the rotation frequency and magnetic field strength (Mestel 1968; Nerney 1980; Nerney \& Suess 1987; Shore \& Brown 1990). It is therefore something of a surprise that we do not see any evidence for so simple a picture among the helium-weak and silicon stars. Our purpose in this study has been to provide better constraints on the properties of the circumstellar environment in order to assist the modeling of the magnetic field and gas distribution and help to understand the origin of the hot plasma.

In an early survey, Brown et al. (1984) observed about a dozen helium-weak and silicon (CP2) stars. They found that there was little evidence for plasmaspheres among the "normal" CP2 stars, but that the sn stars stood out as possessing strong and variable C IV and Si IV resonance lines, a conclusion that was elaborated by Shore et al. (1987) ${ }^{1}$. Brown et al. (1984) attributed the features observed near $\lambda 1550 \AA$ in the non-sn stars in their sample to blends of iron peak elements. However, they had difficulty in interpreting the spectra because they didn't have multiple spectra covering different rotation phases for most of their sample. For instance, one star, HD 142990, a rapidly rotating B3 star, appeared to have a feature at the resonance doublet but this was attributed to Fe III blends.

Our previous study of the helium-weak plasmaspheres (Shore et al. 1990) examined only the sn subsample, finding that HD 5737, 21699, and 79158 all have strong, variable C IV and Si IV, consistent with magnetically controlled circumstellar plasma. There is one peculiarity even in this tiny sample: two of the stars show the same behavior as the helium-strong stars, that is they show plasmaspheres, while one (HD 21699) clearly

\footnotetext{
${ }^{1}$ Throughout this paper, we will refer to the magnetically controlled corotating circumstellar material as the "plasmasphere", following the conventions established in the solar system magnetospheric literature. By this we mean the region of confined, hot plasma embedded within the closed - approximately axisymmetric - global magnetic field whose source of gas is the stellar atmosphere (see e.g., Russell 2001).
}

displays a polar outflow with no strong evidence for a plasmasphere (although it may be present, it doesn't dominate the line profile at any phase). In contrast, Shore \& Brown (1990) showed that all of the helium-strong stars showed the UV signatures of plasmasphere. There are, however, no obvious correlations between any of the stellar properties and the presence or absence of the UV signature of a plasmasphere. HD 79158 and HD 5737, which show essentially the same UV variability, differ considerably in both surface magnetic field strength and rotation frequency while they have approximately the same radius and effective temperature. The $s n$ stars also cohabit the main sequence with many of the stars observed by Brown et al. (1984) that show no compelling evidence for magnetically controlled plasma.

There has been, in the meantime, a substantial increase in the amount of information available on the circumstellar environments of cooler magnetic stars from other wavelength regions. Radio surveys with the VLA (Linsky et al. 1992; Leone et al. 1994; Drake 1998; Trigilio et al. 2000) have detected a number of $6 \mathrm{~cm}$ emitters among the helium-weak stars. Optical studies have also improved, and they now show that variable $\mathrm{H} \alpha$ emission, previously the exclusive property of the helium-strong group, extends to lower effective temperature (Bolton et al. 1986; Bohlender et al. 1992; Bohlender 1994). Among the helium-weak stars, the $\mathrm{H} \alpha$ emission is extremely weak, and it is often discovered only by subtle asymmetries and line profile variations rather than clear emission peaks of the sort seen in the hottest of the helium-strong stars (starting with Walborn 1983; see also Babel 1999). Many of the stars that did not show any dense matter, as derived from UV spectra, still possess strongly emitting circumstellar regions as determined from radio data.

The general properties of the magnetospheric model for the Bp stars have been reviewed by Linsky et al. (1992), Linsky (1993), and Shore (1993, 1999). The plasma is forced to corotate within the Alfvén surface, the radius of which is related to the intrinsic properties of the star through $R_{\mathrm{A}} \sim$ $B^{1 / 3} v_{\infty}^{1 / 6} \dot{M}^{-1 / 6} \Omega^{-1 / 3} R_{\star}$. Here $R_{\star}$ is the stellar radius, $v_{\infty}$ is the terminal velocity of the wind, $B$ is the surface magnetic field strength, $\dot{M}$ is the mass loss rate, and $\Omega$ is the rotation frequency. Linsky et al. (1992), concentrating exclusively on radio measurements, find a general scaling for the intensity of the $6 \mathrm{~cm}$ emission which is approximately of the form $L_{6 \mathrm{~cm}} \sim$ $\dot{M}^{2 / 5} B \Omega^{1 / 3}$. There is considerable uncertainty in the mass loss rates for the cooler (helium-weak) stars compared with the helium-strong stars so their scaling relation is only preliminary. One of the advantages of the UV resonance line measurements is that it provides direct information about the density and velocity of the material in the circumstellar plasma. The strong dependence on the surface magnetic field reflects the nonthermal nature of the emission mechanism, which is likely gyrosynchrotron radiation. The origin of the radio emission may be related to the mass loading of the magnetic field. Havnes \& Goertz (1984) have suggested that the emission is powered by reconnection at the Alfvén surface and that the mass that is stored in the plasmasphere drives the instability. A correlation between the spectroscopic and radio signatures of plasmaspheres/magnetospheres would be expected in this 
case. Usov \& Melrose (1992) invoke current sheet reconnection as the heating mechanism. An alternative mechanism was proposed by Shore et al. (1987) that invokes shear generation of Alfvén waves at the wind-plasmasphere boundary but does not treat the problem of particle acceleration.

In the helium-strong stars, Balmer line emission is modulated with the rotational phase (e.g., Nakajima 1985). The best studied case, HD 37479, is clearly not axisymmetric in either the magnetic or rotational frame (e.g., Groote \& Hunger 1982; Hunger et al. 1990; Hunger \& Groote 1992; Groote \& Hunger 1999). In extending the work to cooler helium-strong and helium-weak stars, Bohlender et al. (1992), and Bohlender (1994) find that a few stars not noted in previous (mainly photographic) studies as having $\mathrm{H} \alpha$ emission actually show it very weakly. In particular, the $s n$-star HD 79158 (=36 Lyn), which shows UV evidence for magnetically controlled circumstellar gas, displays weak profile variations in the core of its $\mathrm{H} \alpha$ line which is likely due to a variable circumstellar absorption component that had been missed in earlier photographic studies. The helium-weak star HD 35502 also displays pronounced emission variability. It therefore seems that many of the hotter members of the helium-weak sequence of chemically peculiar stars possess plasmaspheres. Our goal in this study was to determine the generality of this phenomenon.

\section{Observations}

All of the ultraviolet spectra used in this study are high resolution echelle spectra obtained with the International Ultraviolet Explorer satellite (IUE) using the short wavelength primary (SWP) camera with a resolution of about 10000 . These spectra cover all important resonance lines in the ultraviolet, in particular Si IV $1400 \AA$, C IV $1550 \AA$ À, and Al III $1860 \AA$. Ultraviolet spectra provide a unique spectroscopic signature of a plasmasphere: variations of the C IV and Si IV resonance doublets. Our earlier studies showed that the variations of these lines can be used to obtain the distribution of any magnetically confined plasma, independent of the magnetic field parameters. The profile provides the same information as Doppler imaging methods because the circumstellar plasma is constrained to corotate with the stellar surface.

We obtained observations of the helium-weak stars during 1994 July 1 through July 13. The survey included HD 142301, $142990,144334,144844$, and 146001 . The observing sessions consisted of $4 \mathrm{~h}$ shifts randomly distributed during the two weeks between the first and second half of a normal IUE observing session in an attempt to obtain the broadest possible coverage and to avoid aliasing for the stars for which no periods were known. The sample stars are confirmed members of the Scorpius-Centaurus association (Thompson et al. 1987; de Geus et al. 1989) according to Hipparcos parallaxes. This is useful for comparison with the radio detections, since it means that all of the stars are at approximately the same distance. Association membership should also ensure that the stars are all the same age and had the same initial chemical composition. All IUE spectra for our program stars, and several other helium-weak stars and related objects have been examined. The journal of IUE observations used for the stars in the current study is given in Table 1 . We also list here the synthetic photometric index, $a(\mathrm{C} \mathrm{IV})$ derived as described by Shore et al. (1990) and Shore \& Brown (1990), that measures the equivalent width of the doublet. The typical scatter in these measurements can be assessed from the plots, typically $\pm 0.05(1 \sigma)$ internal and approximately the same for external scatter.

Since earlier studies also seem to show a correlation between the appearance of variable $\mathrm{H} \alpha$ emission, radio emission, and in some cases, ultraviolet signatures of plasmaspheres, we have also obtained phase-resolved spectra of the $\mathrm{H} \alpha$ line of most of the objects observed with the IUE. These data were obtained at two sites: the Canada-France-Hawaii telescope (CFHT) with both the Gecko and $f / 8.2$ coudé spectrographs, and the European Southern Observatory's (ESO) 1.4-m coudé auxiliary telescope (CAT) with the long camera. The CFHT data were acquired over several epochs with the 1872 Reticon and various CCD's, while the ESO observations were obtained remotely from Garching during 1994 May 14-19 with the FA 2048 CCD. The optical data have been processed with IRAF, MIDAS and IHAP reduction softwares. Two points should be noted. First of all, contamination of the $\mathrm{H} \alpha$ profile by telluric lines has been removed where possible (in the case of the ESO measurements, spectra of the rapidly rotating stars HR $5511-\mathrm{A} 0 \mathrm{~V}, v \sin i=$ $351 \mathrm{~km} \mathrm{~s}^{-1}$ - and HR $7446-$ B0.5III, $v \sin i=259 \mathrm{~km} \mathrm{~s}^{-1}$ were taken to identify the telluric lines and measure their strength). Secondly, because of the limited wavelength coverage given by the CFHT Gecko and ESO CAT data there is a very small region of true continuum in the raw data. In some cases this has made precise rectification quite difficult. Despite our best efforts, the continuum chosen for the CFHT data does not always correspond precisely to that of the ESO data. To work around this problem, we have arbitrarily adjusted the continuum level of the CFHT spectra (where needed) so that when overplotted on the ESO data the wings of the lines agree. This correction was always small and on the order of a few per cent of the continuum level. Because of this problem, however, in our discussion of the $\mathrm{H} \alpha$ data given below we will give very little weight to apparent line-depth changes of this order of magnitude. Variations in line shapes should not be affected. Table 2 contains the journal of $\mathrm{H} \alpha$ observations for three stars of particular interest to the discussion below. We have also retrieved the Hipparcos photometry for all the stars discussed in the following sections.

\section{Results}

\subsection{Scorpius-Centaurus association helium-weak stars}

\subsubsection{HD $142301=3 \mathrm{Sco}$}

This is the most extensively studied star in our sample of the Sco-Cen helium-weak stars. Landstreet et al. (1979) give the following ephemeris for this star,

$$
B_{\text {eff }}(\text { Negative Extremum })=\text { JD } 2442921.875( \pm 0.022)
$$

$$
+1.45955( \pm 0.0003) E \text {, }
$$


Table 1. IUE observation journal and C IV measurements for the program helium-weak stars.

\begin{tabular}{|c|c|c|c|c|}
\hline Target & SWP & $\mathrm{JD}(2440000+)$ & $\Phi$ & $a(\mathrm{C}$ IV $)$ \\
\hline \multirow[t]{18}{*}{ HD 142301} & 7899 & 4278.9882 & 0.55 & -0.080 \\
\hline & 21093 & 5596.8431 & 0.45 & -0.314 \\
\hline & 22676 & 5796.2986 & 0.11 & -0.050 \\
\hline & 22681 & 5796.4792 & 0.23 & -0.256 \\
\hline & 23987 & 5961.0250 & 0.97 & -0.019 \\
\hline & 51277 & 9535.0396 & 0.64 & -0.086 \\
\hline & 51291 & 9536.0597 & 0.34 & -0.363 \\
\hline & 51299 & 9536.9896 & 0.98 & -0.047 \\
\hline & 51303 & 9537.1132 & 0.06 & -0.075 \\
\hline & 51312 & 9538.1903 & 0.80 & -0.030 \\
\hline & 51323 & 9539.0924 & 0.42 & -0.234 \\
\hline & 51336 & 9540.1396 & 0.14 & -0.065 \\
\hline & 51352 & 9541.2146 & 0.87 & -0.036 \\
\hline & 51371 & 9542.1167 & 0.49 & -0.370 \\
\hline & 51383 & 9543.2146 & 0.24 & -0.283 \\
\hline & 51400 & 9545.1014 & 0.54 & -0.189 \\
\hline & 51410 & 9545.9806 & 0.86 & -0.052 \\
\hline & 51414 & 9546.1146 & 0.77 & -0.228 \\
\hline \multirow[t]{17}{*}{ HD 142990} & 22195 & 5734.4729 & 0.76 & -0.134 \\
\hline & 22674 & 5796.2417 & 0.85 & -0.050 \\
\hline & 22678 & 5796.3708 & 0.98 & -0.055 \\
\hline & 22682 & 5796.5146 & 0.13 & -0.140 \\
\hline & 51289 & 9535.9903 & 0.54 & -0.156 \\
\hline & 51292 & 9536.0882 & 0.64 & -0.031 \\
\hline & 51301 & 9537.0514 & 0.63 & -0.031 \\
\hline & 51321 & 9539.0201 & 0.64 & -0.046 \\
\hline & 51337 & 9540.1764 & 0.82 & -0.032 \\
\hline & 51339 & 9540.2486 & 0.89 & -0.110 \\
\hline & 51353 & 9541.2493 & 0.91 & -0.091 \\
\hline & 51369 & 9542.0306 & 0.71 & -0.046 \\
\hline & 51370 & 9542.0889 & 0.77 & -0.042 \\
\hline & 51384 & 9543.2438 & 0.95 & -0.209 \\
\hline & 51397 & 9545.0000 & 0.74 & -0.058 \\
\hline & 51401 & 9545.1326 & 0.88 & -0.089 \\
\hline & 51412 & 9546.0458 & 0.81 & -0.111 \\
\hline \multirow[t]{17}{*}{ HD 144334} & 4477 & 3936.9910 & 0.29 & -0.089 \\
\hline & 9224 & 4398.1826 & 0.79 & -0.141 \\
\hline & 21084 & 5595.9472 & 0.98 & -0.018 \\
\hline & 21108 & 5597.9826 & 0.34 & -0.116 \\
\hline & 22675 & 5796.2715 & 0.98 & -0.133 \\
\hline & 22680 & 5796.4431 & 0.09 & -0.027 \\
\hline & 23975 & 5959.9375 & 0.46 & -0.172 \\
\hline & 51290 & 9536.0257 & 0.54 & -0.130 \\
\hline & 51302 & 9537.0806 & 0.24 & -0.031 \\
\hline & 51313 & 9538.2222 & 0.01 & -0.043 \\
\hline & 51320 & 9538.9861 & 0.52 & -0.122 \\
\hline & 51338 & 9540.2125 & 0.34 & -0.068 \\
\hline & 51351 & 9541.1778 & 0.98 & -0.089 \\
\hline & 51368 & 9541.9944 & 0.53 & -0.076 \\
\hline & 51381 & 9543.1493 & 0.30 & -0.108 \\
\hline & 51398 & 9545.0319 & 0.56 & -0.116 \\
\hline & 51411 & 9546.0132 & 0.22 & -0.070 \\
\hline \multirow[t]{4}{*}{ HD 144844} & 51279 & 9535.1153 & & -0.006 \\
\hline & 51322 & 9539.0563 & & -0.013 \\
\hline & 51354 & 9541.2819 & & 0.086 \\
\hline & 51413 & 9546.0826 & & -0.046 \\
\hline \multirow[t]{5}{*}{ HD 146001} & 51278 & 9535.0743 & & 0.000 \\
\hline & 51314 & 9538.2583 & & -0.027 \\
\hline & 51340 & 9540.2806 & & 0.085 \\
\hline & 51382 & 9543.1813 & & 0.015 \\
\hline & 51399 & 9545.0674 & & -0.001 \\
\hline
\end{tabular}

Table 2. Journal of ESO and CFHT $\mathrm{H} \alpha$ spectra of the program helium-weak stars.

\begin{tabular}{|c|c|c|}
\hline Target & Observatory & $\mathrm{JD}(2440000+)$ \\
\hline \multirow[t]{2}{*}{ HD 5737} & CFHT & 8580.815 \\
\hline & CFHT & 9594.027 \\
\hline \multirow[t]{4}{*}{ HD 21699} & CFHT & 8579.874 \\
\hline & CFHT & 8580.863 \\
\hline & CFHT & 8581.828 \\
\hline & CFHT & 8582.815 \\
\hline \multirow[t]{3}{*}{ HD 49333} & CFHT & 8580.099 \\
\hline & CFHT & 8581.058 \\
\hline & CFHT & 8583.068 \\
\hline \multirow[t]{6}{*}{ HD 124224} & CFHT & 9083.992 \\
\hline & CFHT & 9084.076 \\
\hline & ESO & 9487.651 \\
\hline & ESO & 9488.602 \\
\hline & ESO & 9490.643 \\
\hline & CFHT & 9592.732 \\
\hline \multirow[t]{13}{*}{ HD 142301} & CFHT & 9084.033 \\
\hline & CFHT & 9084.111 \\
\hline & ESO & 9487.601 \\
\hline & ESO & 9487.750 \\
\hline & ESO & 9487.855 \\
\hline & ESO & 9488.554 \\
\hline & ESO & 9488.631 \\
\hline & ESO & 9488.788 \\
\hline & ESO & 9488.854 \\
\hline & ESO & 9489.552 \\
\hline & ESO & 9489.687 \\
\hline & ESO & 9489.846 \\
\hline & CFHT & 9592.745 \\
\hline \multirow[t]{7}{*}{ HD 142990} & CFHT & 9084.055 \\
\hline & ESO & 9487.572 \\
\hline & ESO & 9488.680 \\
\hline & ESO & 9489.757 \\
\hline & ESO & 9490.799 \\
\hline & ESO & 9491.803 \\
\hline & CFHT & 9592.778 \\
\hline \multirow[t]{6}{*}{ HD 144334} & ESO & 9487.806 \\
\hline & ESO & 9488.829 \\
\hline & ESO & 9489.639 \\
\hline & ESO & 9489.801 \\
\hline & ESO & 9490.569 \\
\hline & ESO & 9490.686 \\
\hline HD 144844 & ESO & 9487.831 \\
\hline \multirow[t]{3}{*}{ HD 175362} & CFHT & 8579.694 \\
\hline & CFHT & 8580.712 \\
\hline & CFHT & 8581.689 \\
\hline
\end{tabular}

and the comparison with He I $4026 \AA$ photometry by Pedersen \& Thomsen (1977) shows that the helium is weakest at the magnetic poles. The longitudinal magnetic field reverses sign with a positive extremum of $+2900 \mathrm{G}$ and negative extremum of $-1220 \mathrm{G}$. It is a classical helium-weak silicon star and it has an average rotation rate, with $v \sin i$ of $80 \mathrm{~km} \mathrm{~s}^{-1}$. North (1984) quotes a slightly different period, 1.45937 days, but this is inconsistent with our $a(\mathrm{C} I V)$ measurements. Photometric fits to the Hipparcos data gives a period of 1.45955 days, 


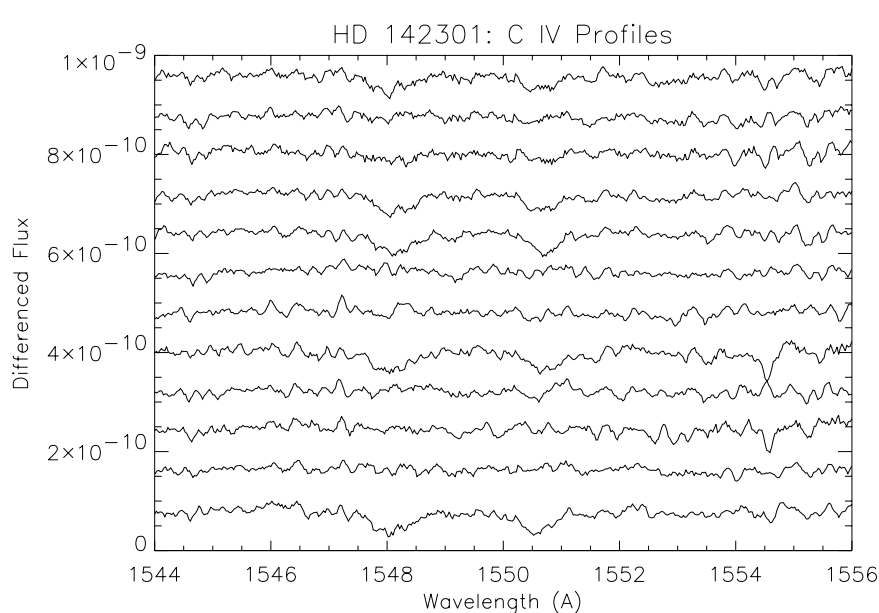

Fig. 1. HD 142301 differenced plot, phased on the ephemeris given in the text.

independently confirming the magnetic results. Borra et al. (1983, hereafter BLT) provide an oblique rotator geometry with $i=30 \pm 10$ and $\beta=78 \pm 8$.

Linsky et al. (1992) report radio measurements at 3.6, 6, and $20 \mathrm{~cm}$ for this star with fluxes of $2.76,2.44$, and $1.81 \mathrm{mJy}$, respectively, on 1988 Oct. 23, and a $6 \mathrm{~cm}$ flux of $4.90 \mathrm{mJy}$ on 1987 July $2 / 3$. They note that the flux on the latter date is variable by a factor of 2 in about $4 \mathrm{~min}$, which may indicate the presence of reconnection events in the plasmasphere and current sheet. Leone et al. (1994, 1996) have also observed the star and also find evidence for variable radio emission.

Figure 1 illustrates the variations in the $\mathrm{C}$ IV resonance line of HD 142301. The error in the published period produces a phase uncertainty of almost $1 / 3$ of a rotation of the star between the latest magnetic field observations and the earliest epoch of multiple IUE observations given in Table 1. However, the IUE data span an interval of more than 5000 days, so we can use these data to improve the period simply plotting the $a(\mathrm{C}$ IV $)$ data for a range of periods close to the published determination. We find the following improved ephemeris (where we follow the normal convention and adopt a magnetic extremum as the zero-point):

$$
\begin{aligned}
B_{\text {eff }}(\text { Negative Extremum })= & \text { JD } 2449545.378( \pm 0.004) \\
& +1.45957( \pm 0.00005) E .
\end{aligned}
$$

The phase uncertainty between the most recent magnetic field data and the earliest IUE $a(\mathrm{CIV})$ indices is now neglible, 0.04 cycles. The magnetic field and $a(\mathrm{CIV})$ data are shown in Figs. 2a,b. We observe that the strongest C IV index occurs at the magnetic equator. The relatively sharp minimum in $a(\mathrm{C}$ IV) variation suggests that the portion of the plasmasphere that is sampled by this technique is narrowly confined to the magnetic equatorial plane. The C IV absorption is very strong at line maximum, in fact the strongest in the sample. Its variability also has the largest amplitude. At minimum strength, the absorption disappears, yet there is no indication of emission seen in some of the helium-strong stars, such as HD 58260 and HD 96446 (cf. Shore \& Brown 1990). The strength of the C IV absorption seems to correlate with the radio data in that HD 142301 is consistently the strongest source.

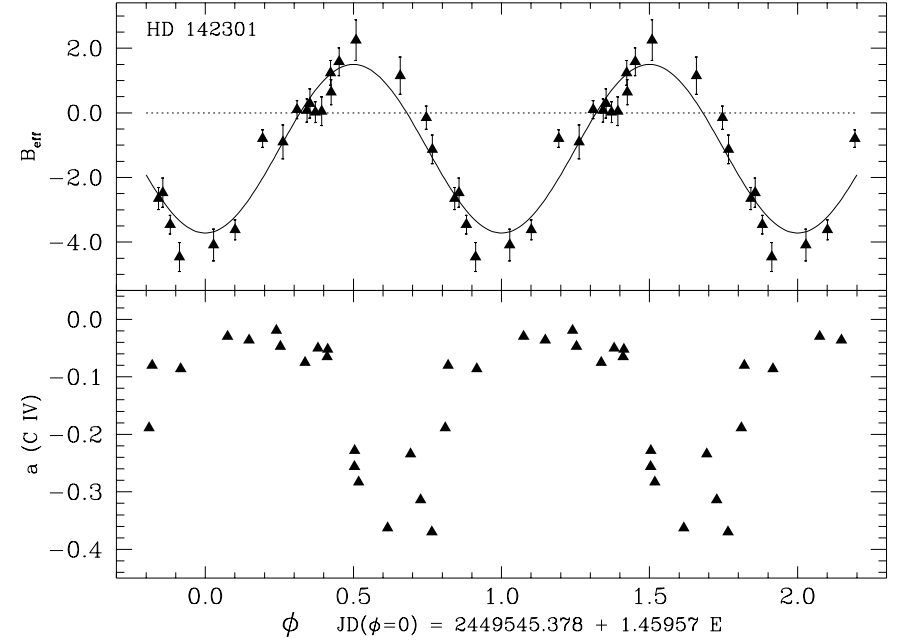

Fig. 2. Magnetic field and IUE $a(\mathrm{C}$ IV) measurements of HD 142301 phased on the new ephemeris given in the text. The strongest C IV index occurs when the equator crosses the line of sight to the observer.

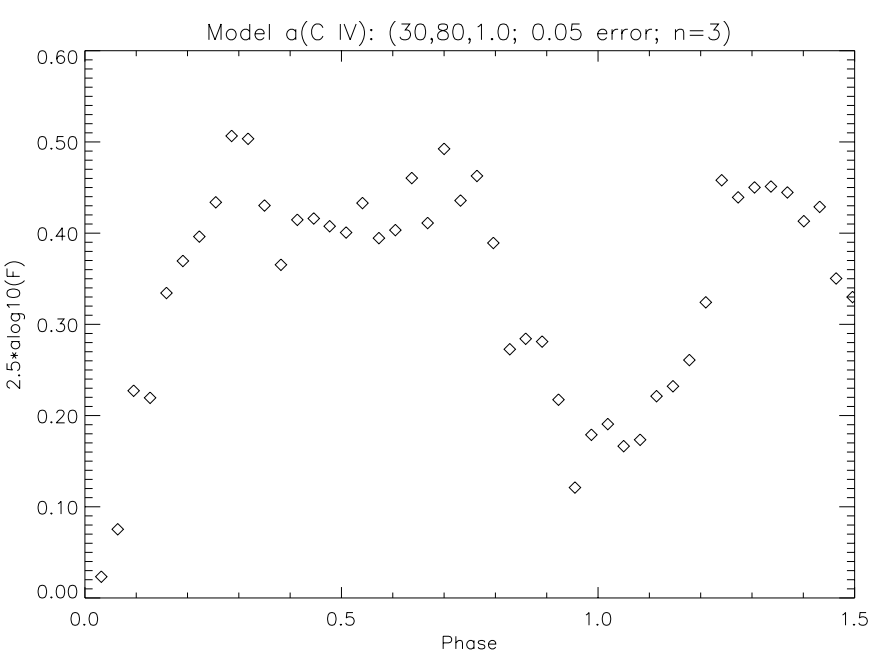

Fig. 3. Oblique rotator model simulation of $a(\mathrm{CIV})$ variations in HD 142301 (see text for description).

We have used the banded oblique rotator model (Shore 1987, 1999; Shore \& Brown 1990) to simulate the variations in HD 142301. This simplified model consists of a reversing layer placed at the magnetic equator and specified by a limb darkening, intensity contrast with the photosphere, flattening (specified by the order for an expansion in Legendre polynomials), and the usual oblique rotator angles $i$, the inclination of the rotation axis to the line of sight, and $\beta$, the obliquity of the magnetic field to the rotational axis. We used values for these parameters obtained from the magnetic data, described earlier in Sect. 3. To simulate the observations, we also included a scatter of 0.05 in $a(\mathrm{C}$ IV) generated by the model. The result is shown in Fig. 3. The model seems in good agreement with the measurements shown in Fig. 1, strengthening our contention that the C IV is formed in an equatorial plasmasphere.

The H $\alpha$ spectra of HD 142301, phased on the above improved ephemeris, are illustrated in Fig. 4. There is no evidence for any form of emission in $\mathrm{H} \alpha$, but the $\mathrm{C}$ II doublet is clearly variable, and strongest when the $a(\mathrm{CIV})$ index is strongest. 


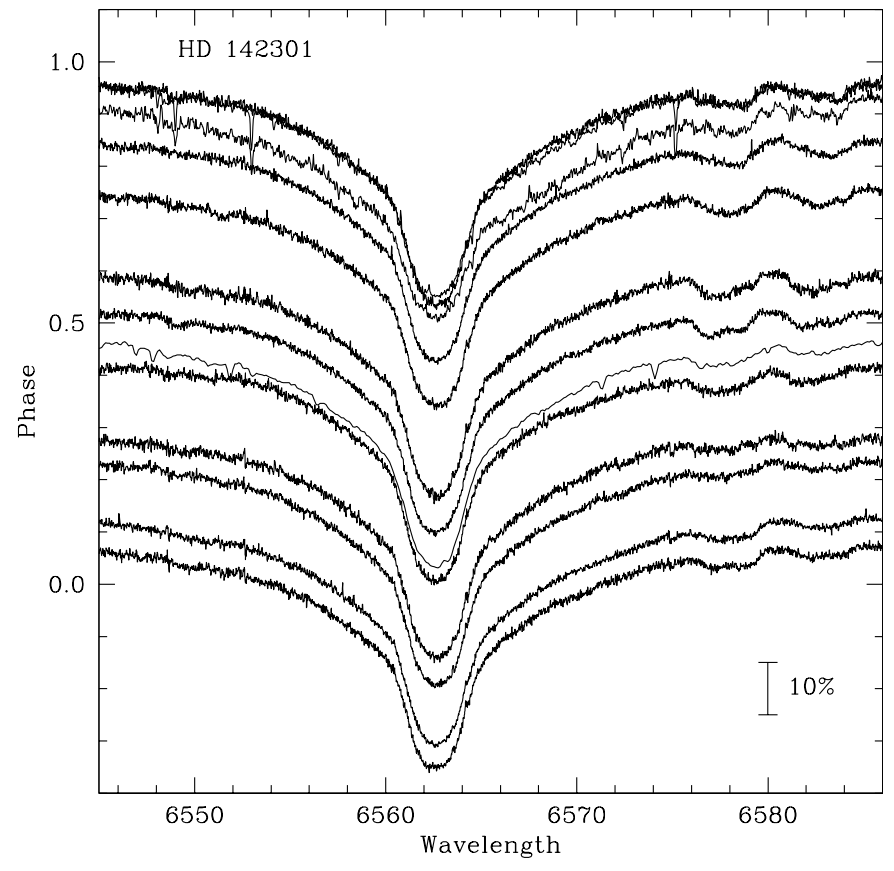

Fig. 4. Observations of the $\mathrm{H} \alpha$ line of HD 142301. Each spectrum has been normalized to a continuum level of 1.0 and the relative intensity scale is shown in the lower right corner. The phase of each observation (obtained with the ephemeris provided in the text) is indicated on the left by locating the continuum of each spectrum at the corresponding phase of the observation. The profile also displays the C II 6578 and 6583 doublet which demonstrates further the comparative stability of the $\mathrm{H} \alpha$ profile.

The lack of $\mathrm{H} \alpha$ emission is perhaps not surprising given the relatively modest $v \sin i$ of the star.

\subsubsection{HD $142990=H R 5942$}

The magnetic field of HD 142990 was first discovered by BLT. They quote two possible periods, $0.492585 \pm 0.000015$ and $0.978430 \pm 0.00008$ days, deriving $i=28 \pm 10$ and $\beta=73 \pm$ 8 for the shorter period and $i \geq 35$ and $\beta \leq 85$ for the longer period. Bohlender et al. (1993) measured the field again with the sole purpose of discriminating between the 0.5 and 1.0 day periods. They succeeded but were still unable to define a unique period. The magnetic field has extrema of +920 and $-1840 \mathrm{G}$ and the field variation is best represented by a period of $0.982920 \pm 0.000020$ days. However, several other periods near this value also fit the magnetic data quite well. Using uvby photometry, Catalano \& Leone (1996) obtain the following ephemeris

$\mathrm{JD}(u v b y$ min. $)=\mathrm{JD} 2442820.017+0.979070( \pm 0.000011) E$.

Since this period is in agreement with one of the alternative periods given by Bohlender et al. (1993) we will adopt it for our discussion below. The Hipparcos catalog gives a period of 0.978987 days, in agreement with Catalano \& Leone's value. Note that even if the 0.982920 day period is the correct choice, the relative phasing of the magnetic field and $a(\mathrm{C}$ IV) curves changes very little.

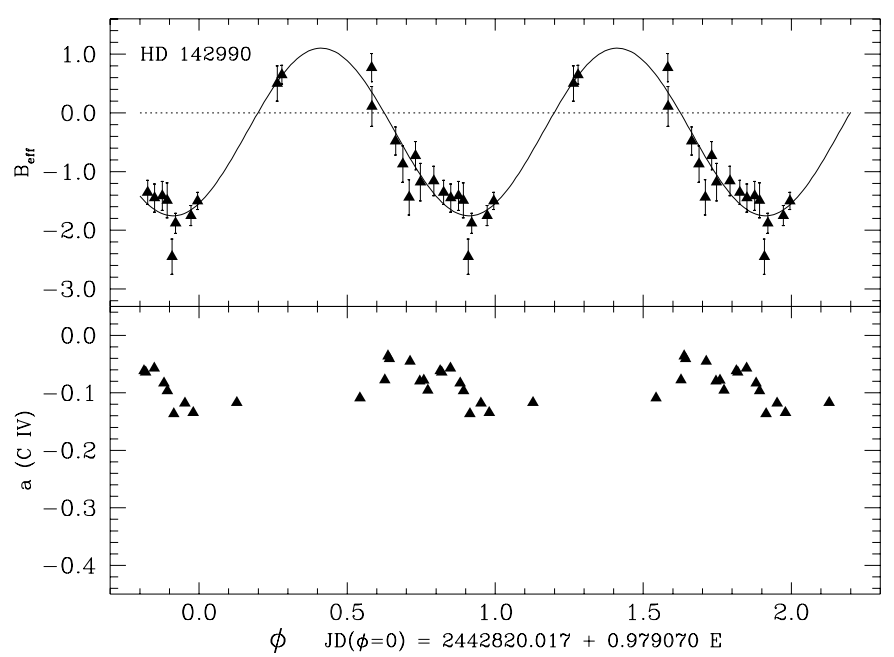

Fig. 5. Magnetic field and IUE $a(\mathrm{C}$ IV) measurements of HD 142990 phased on the ephemeris given in the text. The $a(\mathrm{C}$ IV) measurements have been smoothed to three points with a boxcar average.

Linsky et al. (1992) report radio measurements at 3.6, 6, and $20 \mathrm{~cm}$ for this star with fluxes of $1.98,2.27$, and $<3.3 \mathrm{mJy}$, respectively, and $0.21 \mathrm{mJy}$ for $6 \mathrm{~cm}$ on 1987 July $2 / 3$. They also note that variations of a factor of 4.5 occur on timescales as short as $5 \mathrm{~min}$, much faster than the rotation of the star and possibly due to flaring. Additional measurements by Leone et al. $(1994,1996)$ support the case for radio variability. Leone et al. (1995) have also made observations of the He I 10830 line of HD 142990 to search for the existence of a corona around the star, but no evidence of hot, circumstellar plasma was found.

For HD 142990, the epochs of the magnetic field, photometry, and IUE observations overlap so there is no significant phase uncertainty between the magnetic, photometric, and $a(\mathrm{C}$ IV $)$ data. In light of these data and because of the rather low amplitude of the C IV line profile changes, we have opted not to attempt to improve upon the period adopted above.

The $a(\mathrm{C}$ IV $)$ measurements are not very well distributed in phase, but the apparent minimum coincides with the magnetic equatorial passage. The data are therefore consistent with the C IV variations from a plasmasphere. We have plotted these measurements on the 0.979 day period along with the magnetic field data in Figs. 5a,b to show the phase relationship. The data have been smoothed to 3 points with a boxcar average. The variations seen in the smoothed data match the behavior that is expected for an equatorial plasmasphere, which is antiphase variations of the $a(\mathrm{CIV})$ and the effective magnetic field. The amplitude of the C IV variations for HD 142990 is not as large as for HD 142301, but the magnetic field is either weaker or seen at a slightly different obliquity than for that star. Our oblique rotator models are consistent with the geometry proposed by BLT. There is an indication that the plasmasphere may be slightly more extended in magnetic latitude for this star than for HD 142301.

In Fig. 6 we show the $\mathrm{H} \alpha$ data for HD 142990. In contrast with HD 142301, there is marginal evidence for variability in the $\mathrm{H} \alpha$ profile of HD 142990. Small emission "bumps" appear between phases 0.2 and 0.3 located approximately 


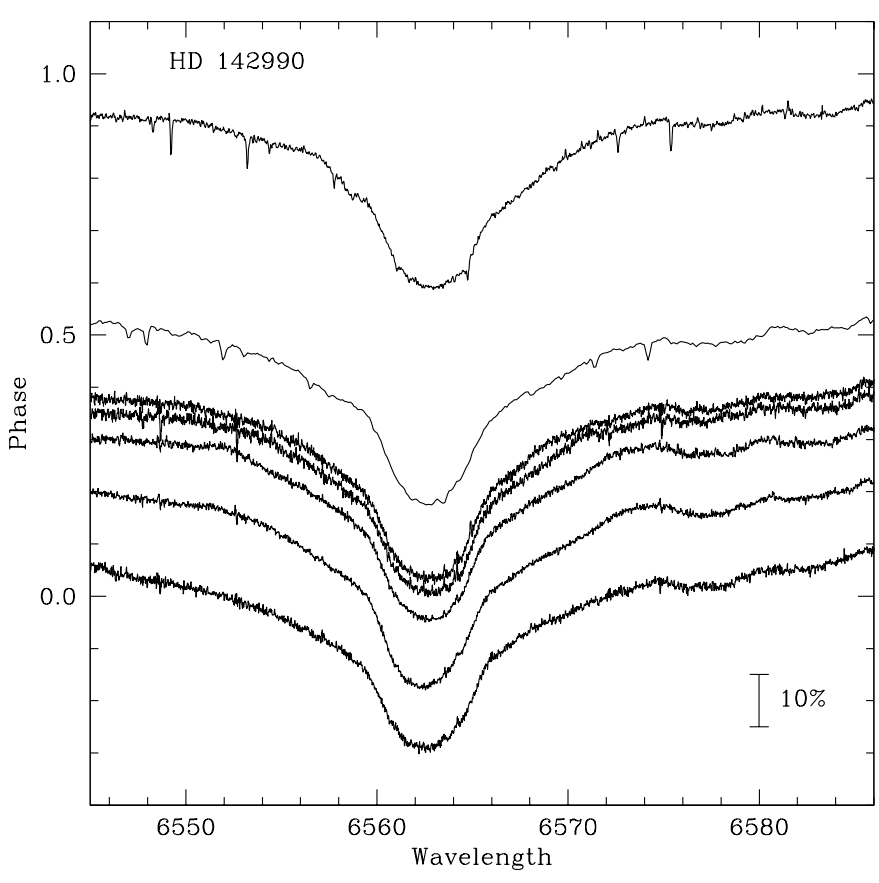

Fig. 6. Observations of the $\mathrm{H} \alpha$ line of HD 142990 (see Fig. 4).

$\pm 480 \mathrm{~km} \mathrm{~s}^{-1}$ from the line center. The emission is most prominent on the blue wing of the line but careful examination of the figure suggests a similar effect in the red wing. Since the feature appears at the same absolute velocity relative to line center, it is not likely an instrumental artifact. There is a hint of a slight asymmetry in the line core near 0.35 (compare to the profile near phase 0.05 ), but this could be caused by variations in the surface helium abundance of the star. Clearly additional $\mathrm{H} \alpha$ observations would be of interest. The fact that HD 142990 has a more rapid rotation rate but a weaker magnetic field than HD 142301 hints at the possible role of a centrifugally driven wind in producing a dense plasmasphere.

\subsubsection{HD $144334=\operatorname{HR} 5988$}

For HD 144334, BLT found a reversing field and quoted what they thought was a unique period of 3.610 days. They were unable to determine oblique rotator parameters unambiguously from the available data, but presented arguments for either large obliquity or high inclination for the rotation axis. Photometry by North \& Waelkens (1983), North (1987), and Catalano \& Leone (1996) yields a period of 1.494971 days. In disagreement with BLT, we find that the magnetic field data are also reasonably well described by such a period, although the $\chi^{2} / n$ fit is 1.63 versus 0.71 for the 3.61 day period of BLT. The larger value of $\chi^{2} / n$ is, however, typical of the fit to a sinusoid of other magnetic stars. The best fit sinusoid yields magnetic extrema of +320 and $-1240 \mathrm{G}$. We therefore adopt the ephemeris of North (1987), based on the Geneva [U] magnitude $\left(U_{\mathrm{Gen}}\right.$ max. refers to the maximum value of the fundamental component of the Fourier series fitted to the light flux in this band):

$\mathrm{JD}\left(U_{\mathrm{Gen}} \max .\right)=\mathrm{JD} 2445229.708+1.494971( \pm 0.000024) E$.

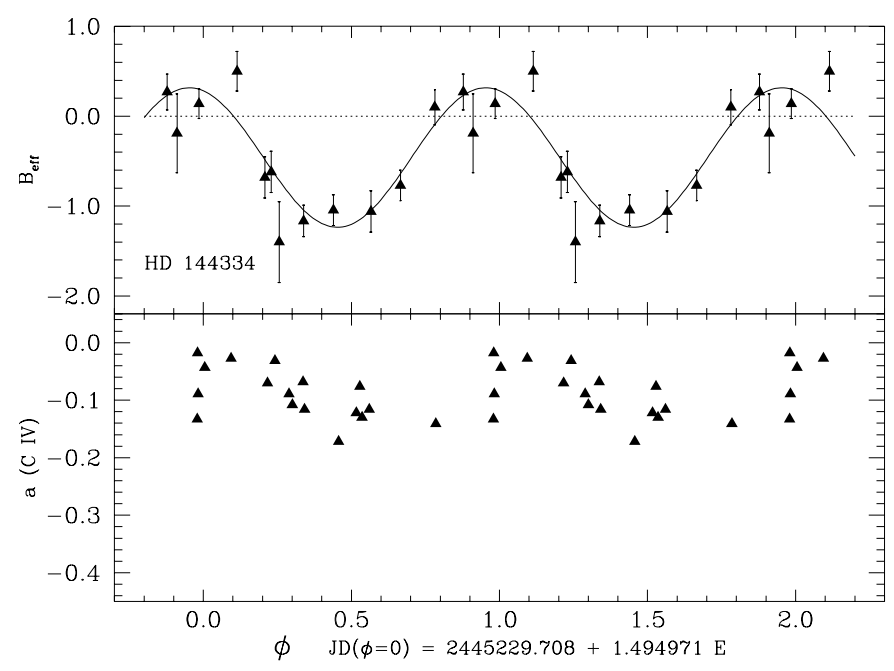

Fig. 7. Magnetic field and IUE $a(\mathrm{C}$ IV) measurements of HD 144334 phased on the ephemeris given in the text. The $a(\mathrm{C}$ IV) measurements have been smoothed to three points with a boxcar average.

The Hipparcos catalog gives a period of 1.49498 days, in excellent agreement with the groundbased photometry. We also note that the Hipparcos band light curve is nearly identical to that of HD 142990.

Linsky et al. (1992) found only weak radio emission. They report only $6 \mathrm{~cm}$ detections for this star, $0.4 \mathrm{mJy}$ on 1988 Oct. 23 and $0.24 \mathrm{mJy}$ on 1988 Oct. 28 . They give only an upper limit of $0.8 \mathrm{mJy}$ at $20 \mathrm{~cm}$ on 1988 Oct. 23. Leone et al. (1994) did not detect the star at $6 \mathrm{~cm}$ in 1992. Drake et al. (1994) report the marginal detection of this star with the PSPC on ROSAT from All-sky Survey data, $(3.7 \pm 1.3) \times 10^{-2} \mathrm{~s}^{-1}$. Only this star and HD 146001 have been detected among the helium-weak stars in our survey.

The present CIV measurements, illustrated in Figs. 7a,b compared with the magnetic field measurements, show some slight enhancement of the doublet. When plotted on the above period, there may be a low amplitude variation in the $a(\mathrm{C}$ IV) index. What we can say is that the line is never as strong as the C IV seen for HD 5737, 21699, 79158, or 142301, and is usually about the same strength as we observe in HD 142990.

Figure 9 shows that there is no evidence for emission or line asymmetry in $\mathrm{H} \alpha$. We also note the strong variations in the C II doublet. Particularly interesting is the change in appearance of the line from a relatively sharp feature near phase 0.5 to a considerably broader and weaker profile near phase 0.1 . This variability likely explains the range of $v \sin i$ (from 44 to $140 \mathrm{~km} \mathrm{~s}^{-1}$ ) quoted in the literature and first pointed out by BLT. Although beyond the scope of this paper, in light of these large spectrum variations, an analysis of the elemental distribution of HD 144334 would be important.

\subsubsection{HD 144844}

BLT do not detect a field for this star, although they made a large number of observations. Our CIV measurements are consistent with this. There is no evidence for hot plasma around this star on the basis of the UV observations. 


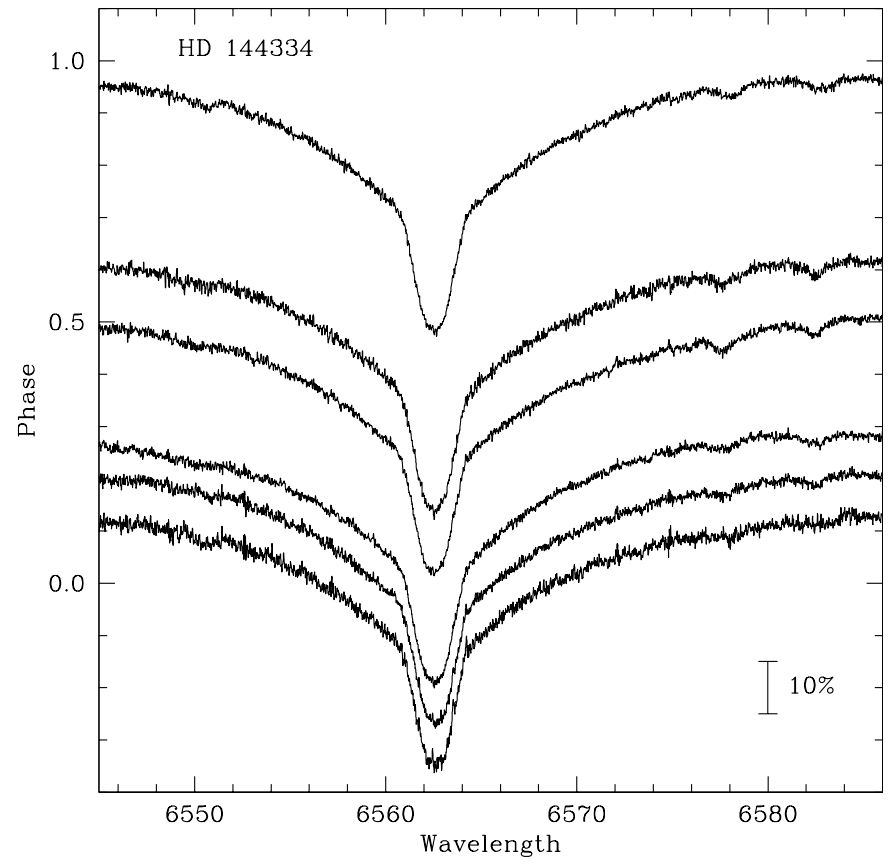

Fig. 8. Observations of the $\mathrm{H} \alpha$ line of HD 144334 phased on the rotation period of the star (see text and Fig. 4). No variable $\mathrm{H} \alpha$ emission or absorption is apparent.

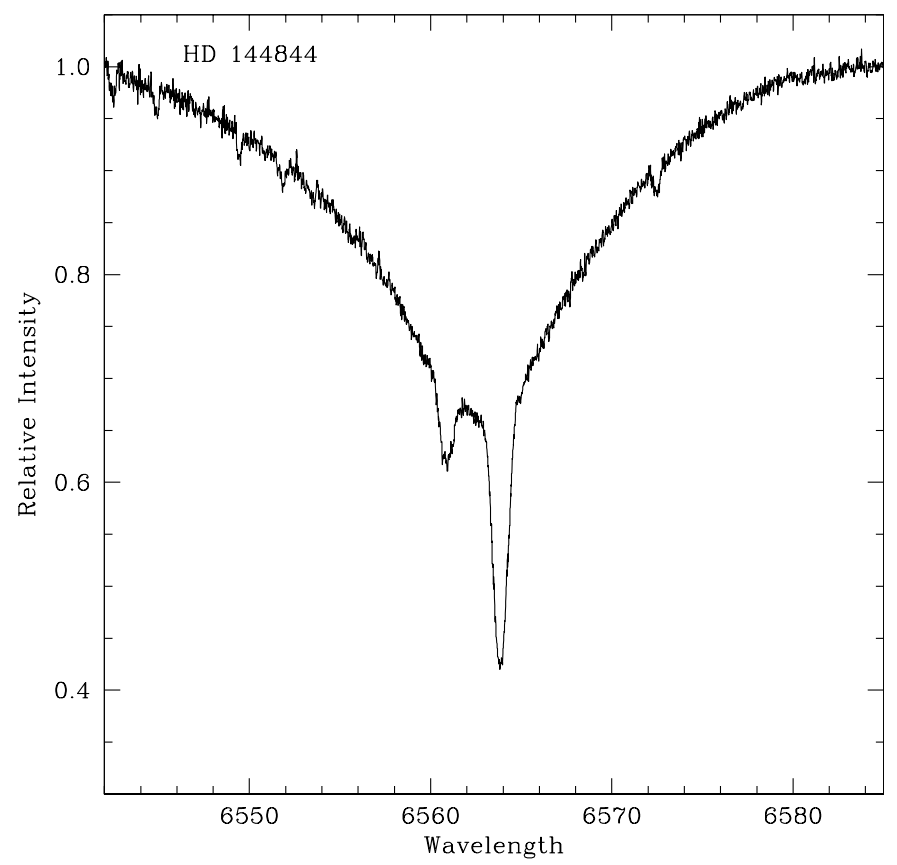

Fig. 9. Observation of the $\mathrm{H} \alpha$ line of HD 144844 (see Fig. 4).

Linsky et al. (1992) report a marginal detection of $6 \mathrm{~cm}$ with a flux of 0.19 mJy on 1988 Oct. 23 and only upper limits of $<0.20,<0.18$, and $<0.49 \mathrm{mJy}$ at $3.4,6$, and $20 \mathrm{~cm}$ on 1988 Oct. 28. Drake et al. (1994) report only upper limits to the radio emission. Not surprisingly, no emission is apparent in our single $\mathrm{H} \alpha$ spectrum of the double-line spectroscopic binary (Hoffleit \& Jaschek 1991).



Fig. 10. Observations of the $\mathrm{H} \alpha$ line of HD 124224 phased on the rotation period of the star (see text and Fig. 4). Any variation of the line profile appears to be quite subtle.

\subsubsection{HD $146001=H R 6054$}

Neither Pedersen \& Thomsen (1977) nor BLT find this star to be a variable. HD 146001 is classified as helium-weak by Nissen (1974) but BLT failed to detect a magnetic field at the $2.3 \sigma$ level. Linsky et al. (1992) report a detection at $6 \mathrm{~cm}$ on 1988 Oct. 23 with a flux of $0.32 \mathrm{mJy}$, but only upper limits of $<0.22,<0.25$, and $<0.83 \mathrm{mJy}$ at $3.4,6$, and $20 \mathrm{~cm}$ on 1988 Oct. 28. Drake et al. (1994) report a marginal PSPC detection with ROSAT from All-sky Survey data. They found a count rate of $(4.5 \pm 1.8) \times 10^{-2} \mathrm{~s}^{-1}$ in only the soft band. Drake et al. suggest that this star may be a binary and that this is the explanation for the X-rays. Leone et al. (1995) find no peculiarity in the He I 10830 line. Hipparcos photometry shows a variation of less than 0.002 with only a few deviant points $(>3 \sigma)$ and is insufficient to determine any periodicity. Our IUE spectra show no evidence for enhanced C IV absorption.

\subsection{Other helium-weak stars}

\subsubsection{HD $124224=$ CU Vir}

This rapid rotator shows no evidence in 12 archival spectra for either variability of the C IV profile or for any enhanced absorption at $1500 \AA$; this spectral region is consistent with rotationally broadened Fe II and Fe III lines (see Brown et al. 1985 for discussion). Some variability is apparent in the $\mathrm{H} \alpha$ profile of the star, shown in Fig. 10 where we have phased the $\mathrm{H} \alpha$ observations on the ephemeris of Pyper et al. (1998):

$\mathrm{JD}(B$ light max. $)=\mathrm{JD} 2435178.9025+0.52070308 E$.

While the phase coverage is obviously very poor, the line clearly becomes asymmetrical near phase 0.6 . This is most 


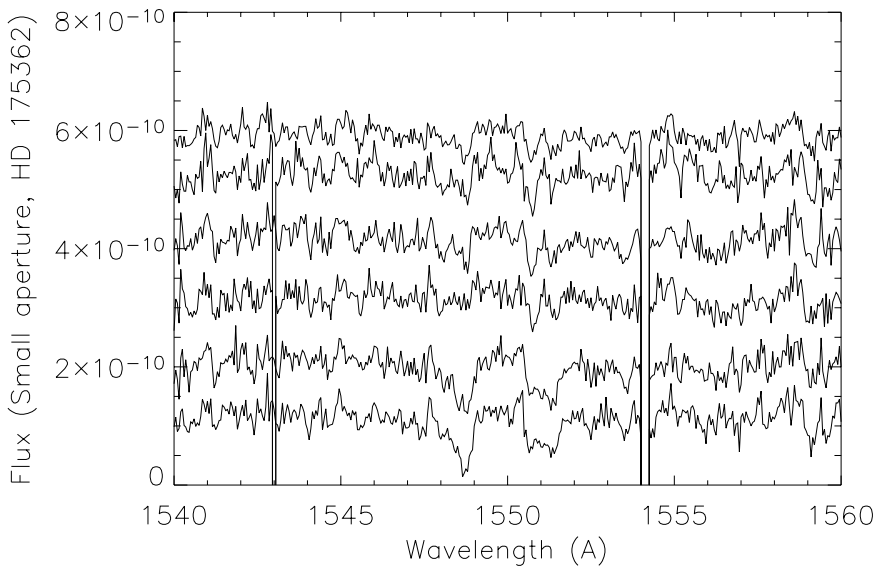

Fig. 11. Sequence of CIV variations in HD 175362. From bottom to top, with phases in parentheses: SWP 3065 (0!64), 3066 (0?65), $3090(0.10), 3098(0.23), 3126(0.80)$, and $3242(0.54)$. Phase $0^{\mathrm{p}} 0$ corresponds to $B_{\text {eff }}$ extremum (negative).

likely not a result of filling in of the profile by circumstellar emission, but rather a result of the large variation in the helium abundance over the star's surface. As Pyper et al. (1998) demonstrate in their Fig. 5, the He I equivalent widths of HD 124224 vary by a factor of more than four during the rotation period. Unfortunately it is very difficult to judge the relative phasing our $\mathrm{H} \alpha$ observations and their helium equivalent width data since this star apparently experienced an abrupt change in its rotational period at some time between 1983 and 1987 (Pyper et al. 1998). Additional $\mathrm{H} \alpha$ observations of HD 124224 would clearly be of interest.

Leone et al. $(1994,1996)$ report radio fluxes of 4.3, 4.6, 3.8, and $2.6 \mathrm{mJy}$ at $1.3,2,6$, and $20 \mathrm{~cm}$ respectively, which makes HD 124224 one of the strongest radio sources among the magnetic peculiar stars. More recently, Trigilio et al. (2000) have reported $1.4,5,8.4$, and $15 \mathrm{GHz}$ coherent radio emission from this star which is almost $100 \%$ polarized (right circular) with the maximum emission occurring at magnetic equatorial crossing. They interpret this effect as emission by electrons accelerated in an equatorial current sheet mirroring from an asymmetric magnetic field at the poles.

\subsubsection{HD $175362=H R 7129$}

This is one of the hottest helium-weak stars and also one of the most extensively studied (see BLT; Bohlender et al. 1987; Mathys 1991). No clear variation is seen from the large aperture spectra; the C IV profile is generally weak and dominated by Fe II and Fe III absorption. An early spectral sequence, however, was obtained with the small aperture during 1978 and these show one pair of successive spectra (Fig. 11) at a strong line phase. Consequently, we include this star among the detected plasmaspheres. The pair of spectra agree with the phase of magnetic equatorial crossing, 0 p 65 , while the rest of the spectra were generally obtained at phases closer to magnetic polar traversals. Only upper limits are given for the radio flux (Leone et al. 1996) and three CFHT spectra obtained on successive nights show no $\mathrm{H} \alpha$ variability, but very large variations in the lines of other elements.

\subsubsection{Other objects}

Small samples of archival data for HD 28843 and HD 49333 were also examined. There is no evidence for variability in the HD 28843 data, which has also been discussed by Shore et al. (1987). A photometric period of 1.374 days has been derived by Pedersen \& Thomsen (1977), but there is no support for this from the ultraviolet data. Catalano \& Leone (1996) quote a period of 2.179232 days for HD 49333. Here there is evidence for some very slight spectrum variability, but not of C IV. The silicon lines, especially Si II 1260, $1264 \AA$ and Si II $1533 \AA$ appear to vary slightly. The Al III $1860 \AA$ doublet, the Si IV $1400 \AA$ doublet, and the C IV $1550 \AA$ lines are stable to within the accuracy of the SWP. There is a possible change at C IV $1548 \AA$ but this is very questionable. In neither case is the archival data set sufficient to place strong limits on the variability, but the lines are certainly never as strong as we observe in either HD 142301 or HD 142990. H $\alpha$ spectra we have obtained for HD 49333 show no evidence for variability.

Three other helium-weak stars, HD 5737, 21699, and 79158, have already been discussed at length in separate papers (Brown et al. 1985; Shore et al. 1987, 1990). All three display large amplitude C IV and Si IV variations, and all three show saturated resonance line profiles at their respective line maxima. Only HD 142301, among the present sample, shows similar line variations. However, neither HD 5737 or HD 21699 have been detected at centimeter radio wavelengths (Linsky et al. 1992). They also reported a null detection for HD 79158, but Drake (1997, private communication) detected a $0.45 \pm$ $0.05 \mathrm{mJy} 6 \mathrm{~cm}$ flux on 22 May 1994. These three stars are moderately slow rotators, spanning the range of the group detected in Sco-Cen. Yet again, there is no obvious correlation with any intrinsic stellar property. The anomaly in this group is that HD 21699 appears to show its strongest C IV absorption coincident with the magnetic pole, not the equator as observed in all of the other stars and also for the helium-strong stars. This may be a problem with the period, but the magnetic and IUE data discussed by Brown et al. (1985) were nearly simultaneous and the period was independently determined photometrically ${ }^{2}$. We have already mentioned the $\mathrm{H} \alpha$ variability of HD 79158 above; neither HD 5737 nor HD 21699 shows any sign of variability in this line.

\section{Discussion}

HD 142301, HD 142990, HD 144334, and HD 175362 are newly detected magnetospheric variables. None of the other helium-weak stars in our Sco-Cen sample nor the other heliumweak stars in the IUE archives show this behavior except for the $s n$ subgroup. To substantiate the anomalous nature of the

\footnotetext{
2 As discussed in Shore \& Brown (1990), however, polar outflow is required to model the C IV profiles in several helium-strong stars, notably HD 94664, that appear to be aligned rotators viewed rotation pole-on.
} 

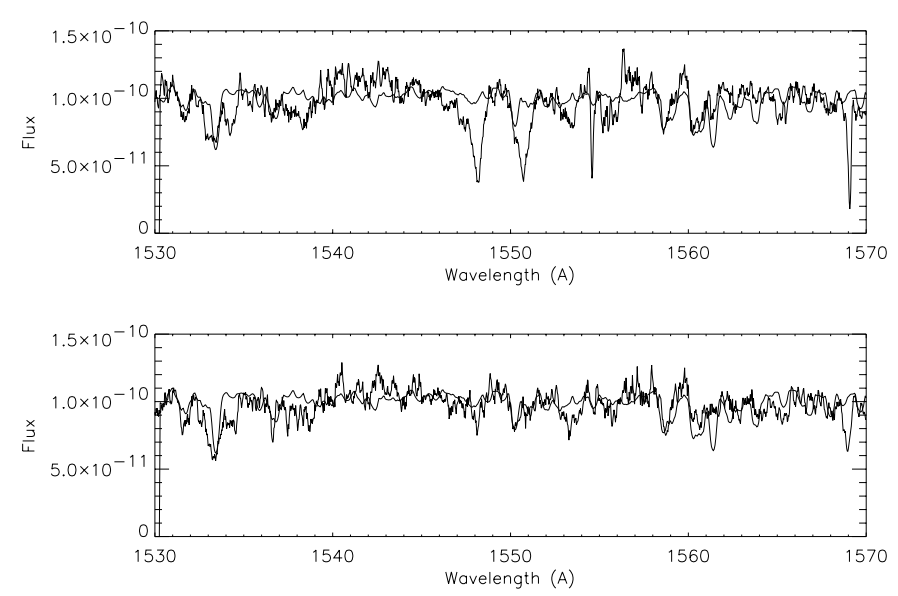

Fig. 12. Comparison between IUE observations of HD 142301 at the two extreme phases for C IV line strength with stellar atmospheres model calculation (see text for details).

C IV line, we have computed a set of model spectra using the PHOENIX model atmosphere code (see Aufdenberg et al. 1998). The spectra are fully line blanketed, and for purposes of this comparison we have used only an LTE grid. In Fig. 12 we show a comparison of the $14454 \mathrm{~K}$ model (using the gravity and temperature from Andersen 1991), with rotational broadening of $50 \mathrm{~km} \mathrm{~s}^{-1}$. This provides the best overall fit to the absorption line spectrum other than the CIV line. We compare in the figure the model with the two extreme phases of HD 142301, which has the strongest C IV variations in our sample. Notice that part of the broadening of the $1550 \AA$ component of the C IV doublet is due to photospheric absorption by Fe II and Fe III, which is the only residual absorption at C IV minimum. The lack of variation of any other lines in this portion of the spectrum has been noted previously in other papers in this series, notably for HD 21699 by Brown et al. (1985). The improved atmospheres reinforce our original conclusions.

What clues do we now possess for the origin of the observed magnetospheric plasma and nonthermal phenomena in the helium-weak stars? At first glance, it appears to be merely a catalog of negatives. There is no strong correlation of the C IV excess with integrated surface magnetic field strength: HD 5737 has a barely detectable field while HD 142301 is among the strongest. It does not strongly correlate with rotation period: HD 124224 and HD 142990, with periods of about 1/2 day, have no, or weaker, C IV absorption at all phases than HD 5737, which has a period of about 24 days. The C IV absorption appears to be independent of the degree of helium weakness; the initial uniqueness of the $s n$ subgroup has vanished with these new detections. The stellar masses are similar, their radii are similar, and the fact that we have three new stars in the Sco-Cen association while we have failed to detect several others in the same association indicates that it is not initial chemical composition or evolutionary status.

An indication that something positive may have emerged from this sample is that the presence of a detectable magnetic field does seem to be required for the C IV absorption to be detected. In addition, there may be a lower effective temperature cutoff to the phenomenon, but this could simply be the disappearance of the searched-for signature due to radiative transfer effects rather than the actual absence of any trapped circumstellar plasma. The radio flux and X-ray fluxes serve only as fair predictors of the detectability of enhanced C IV absorption. The strongest radio source, HD 142301, has the strongest C IV doublet while the weakest detected source, HD 144334, may show some C IV enhancement but does not show obvious variability. Yet the radio source HD 124224 shows no C IV variability (and no enhanced absorption at any observed phase), while HD 175362 displays a plasmasphere yet no detectable radio emission.

The helium-weak stars represent the cool extension of the helium-strong stars. The discovery of the C IV and X-ray variability of the extremely young $\mathrm{O}$-star $\theta^{1}$ Ori $\mathrm{C}$ extends the same behavior to the hottest main sequence stars (see Donati et al. 2002, and references therein). For this star, the magnetospheric heating has a more obvious source, a radiatively driven stellar wind that supplies the plasma as a trapped shocked structure and also provides a source for Alfvén wave heating through shear at the boundary between the closed and open field lines. However, this mechanism is not readily applicable to the helium-weak stars for which mass loss is expected to be quite low. The structure of the plasmasphere should be similar to, but more extreme than, Jupiter (Dressler 1983; Russell 2001), even in the absence of external forcing by the solar wind. The oblique rotator maintains approximate corotation to the Alfvén surface, and inertial effects concentrate matter into a disk in the magnetic equatorial plane. Because this does not coincide with the rotational equator, the plasma is forced into a periodic oscillation on the rotational timescale. In addition, the Alfvén radius is smaller for this region because of the increased density so the sheet may be corrugated and not strictly corotating. For planetary magnetospheres, which are strongly interacting with the solar wind, this produces reconnection in the magnetotail with attendant acceleration of electrons. It is reasonable to expect that reconnection may be occurring within the magnetospheric gas, leading to sporadic variations of the radio emission and a source for local heating. This picture adopted to explain both the radio emission and flaring - must, however, not tie directly to the inner plasmasphere we observe with the UV resonance lines but could serve as the source for the heating required for the plasma. In particular, the backward flowing particles will dissipate their energy in the stellar atmosphere. This could produce the analog of the auroral ring, a region in a planetary atmosphere that is strongly heated. If this occurs, it would be a natural way to load the plasmasphere with gas. At present, however, this remains a speculation.

Note added in proofs: Subsequent to the final submission of this paper, two studies of magnetosphere/plasmasphere models have appeared: Preuss, O., Schüssler, M., Holzwarth, V., \& Solanki, S. K. 2004, A\&A, 417, 987 and Trigilio, C., Leto, P., Umana, G., Leone, F., \& Buemi, C. S. 2004, A\&A, 418, 593.

Acknowledgements. This research was supported by the guest IUE observer program through NASA grant NAG 5-2186. C.T.B. is supported by a Discovery grant from the Natural Sciences and 
Engineering Research Council of Canada. We wish to thank Doug Brown, Steve Drake, Ivan Hubeny, John Landstreet, Ted LaRosa, Jeff Linsky, Gauthier Mathys, Francesco Pegoraro, and Ian Short for valuable discussions. We especially thank Peter Hauschildt and Jason Aufdenberg for supplying the PHOENIX models and for many illuminating discussions, and we thank the referee for valuable questions and suggestions. During our observing program, the staff of the IUE observatory took great pains to insure its proper scheduling and execution; this program, begun in 1980 to survey the variations of the helium peculiar stars, would never have been possible without the flexibility of this satellite and we dedicate this study to its memory.

\section{References}

Andersen, J. 1991, A\&AR, 3, 91

Aufdenberg, J. P., Hauschildt, P. H., Shore, S. N., \& Baron, E. 1998, ApJ, 498, 837

Babel, J. 1999, in Variable and Non-spherical Stellar Winds in Luminous Hot Stars, ed. B. Wolf, O. Stahl, \& A. W. Fullerton (Berlin: Springer-Verlag), 187

Barker, P. L., Brown, D. N., Bolton, C. T., \& Landstreet, J. D. 1982, in Advances in Ultraviolet Astronomy: Four Years of IUE Research, ed. Y. Kondo, J. Mead, \& R. D. Chapman (NASA CP-2238), 589

Bohlender, D. A. 1994, in Pulsation, Rotation and Mass Loss in Early-Type Stars, ed. L. A. Balona, H. F. Henrichs, \& J. M. Le Contel (Dordrecht: Kluwer), 155

Bohlender, D. A., Bolton, C. T., \& Walker, G. A. H. 1992, in Atmospheres of Early-type Stars, ed. U. Heber, \& C. S. Jeffrey (Berlin: Springer-Verlag), 221

Bohlender, D. A., Landstreet, J. D., \& Thompson, I. B. 1993, A\&A, 269, 355

Bohlender, D. A., Landstreet, J. D., Brown, D. N., \& Thompson, I. B. 1987, ApJ, 323, 325

Bolton, C. T. 1984, Hvar Obs. Bull., 7, 241

Bolton, C. T., Fullerton, A. W., Bohlender, D. A., Landstreet, J. D., \& Gies, D. 1986, in Physics of Be Stars, ed. A. Slettebak, \& T. P. Snow (Cambridge: Cambridge University Press), 82

Bolton, C. T., Harmanec, P., Lyons, R. W., Odell, A. P., \& Pyper, D. M. 1998, A\&A, 337, 183

Bolton, C. T., \& Short, I. 1994, in Pulsation, Rotation and Mass Loss in Early-Type Stars, ed. L. A. Balona, H. F. Henrichs, \& J. M. Le Contel (Dordrecht: Kluwer), 171

Borra, E. F., Landstreet, J. D., \& Thompson, I. 1983, ApJS, 53, 151

Brown, D. N., Shore, S. N., \& Sonneborn, G. 1985, AJ, 90, 1354

Brown, D. N., Shore, S. N., Barker, P. K., \& Sonneborn, G. 1984, in The future of ultraviolet astronomy based on six years of IUE research, ed. J. Mead, R. D. Chapman, \& Y. Kondo (NASA CP-2349), 487

Catalano, F. A., \& Leone, F. 1996, A\&A, 311, 230

de Gues, E. J., de Zeeuw, P. T., \& Lub, J. 1989, A\&A, 216, 44

Dessler, A. J. 1983, Physics of the Jovian magnetosphere (Cambridge: Cambridge Univ. Press)

Donati, J.-F., Babel, J., Harries, T. J., et al. 2002, MNRAS, 326, 1265

Drake, S. A. 1998 , CoSka, 27, 382

Drake, S. A., Abbott, D. A., Bastien, T. S., et al. 1987, ApJ, 322, 902

Drake, S. A., Linsky, J. L., \& Bookbinder, J. A. 1994, AJ, 108, 2203

Drake, S. A., Linsky, J. L., Schmitt, J. H. M. M., \& Russo, C. 1994, ApJ, 420, 387a
Groote, D., \& Hunger, K. 1976, A\&A, 52, 303

Groote, D., \& Hunger, K. 1982, A\&A, 116, 64

Groote, D., \& Hunger, K. 1999, A\&A, 351, 554

Havnes, O., \& Goertz, C. K. 1984, A\&A, 138, 421

Hoffleit, D., \& Warren, Jr., W. H. 1991, The Bright star catalogue, 5th edition

Hunger, K., \& Groote, D. 1992, in Peculiar versus normal phenomena in A-type and related stars, ed. F. Castelli, \& M. M. Dworetsky (San Francisco), ASP Conf. Ser., 394

Hunger, K., Heber, U., \& Groote, D. 1990, in Hot luminous stars, ed. C. Garmany (San Francisco), ASP Conf. Ser., 307

Landstreet, J. D., Borra, E. F., \& Fontaine, G. 1979, MNRAS, 188, 609

Leone, F., Lanzafame, A. C., \& Pasquini, L. 1995, A\&A, 293, 457

Leone, F., Trigilio, C., \& Umana, G. 1994, A\&A, 283, 908

Leone, F., \& Umana, G. 1993, in Peculiar versus normal phenomena in A-type and related stars, ed. F. Castelli, \& M. M. Dworetsky (San Francisco), ASP Conf. Ser., 541

Leone, F., Umana, G., \& Trigilio, C. 1996, A\&A, 310, 271

Linsky, J. L. 1993, in Peculiar versus normal phenomena in A-type and related stars, ed. F. Castelli, \& M. M. Dworetsky (San Francisco), ASP Conf. Ser., 507

Linsky, J. L., Drake, S. A., \& Bastien, T. S. 1992, ApJ, 393, 341

Mathys, G. 1991, A\&A, 89, 121

Mestel, L. 1968, MNRAS, 138, 359

Nakajima, R. 1985, Ap\&SS, 116, 285

Nerney, S. 1980, ApJ, 242, 723

Nerney, S., \& Suess, S. 1987, ApJ, 321, 355

Nissen, P. E. 1974, A\&A, 36, 57

North, P. 1984, A\&AS, 55, 259

North, P. 1987, A\&AS, 69, 371

North, P., \& Waelkens, C. 1983, IBVS, 2372

Pedersen, H., \& Thomsen, B. 1977, A\&A, 30, 11

Pyper, D. M., Ryabchikova, T., Malanushenko, V., et al. 1998, A\&A, 339,822

Russell, C. T. 2001, Planet. Space Sci., 49, 1005

Shore, S. N. 1987, AJ, 94, 731

Shore, S. N. 1990, in Evolution in Astrophysics: IUE in the Era of new space missions, ed. E. Rolfs (ESA SP-316)

Shore, S. N. 1993, in Peculiar versus normal phenomena in A-type and related stars, ed. F. Castelli, \& M. M. Dworetsky (San Francisco), ASP Conf. Ser., 528

Shore, S. N. 1999 in Variable and non-spherical stellar winds in luminous hot stars, ed. B. Wolf, O. Stahl, \& A. W. Fullerton (Berlin: Springer-Verlag), 178

Shore, S. N., \& Brown, D. N. 1990, ApJ, 365, 665

Shore, S. N., Brown, D. N., \& Sonneborn, G. 1987, AJ, 94, 737

Shore, S. N., Brown, D. N., Sonneborn, G., Landstreet, J. D., \& Bohlender, D. A. 1990, ApJ, 348, 242

Thompson, I., Brown, D. N., \& Landstreet, J. D. 1987, ApJS, 64, 219

Trigilio, C., Leto, P., Leone, F., Umana, G., \& Buemi, C. 2000, A\&A, 362,281

Usov, V. V., \& Melrose, D. B. 1992, ApJ, 395, 575

Walborn, N. R. 1983, ApJ, 268, 195

Vauclair, S. 1975, A\&A, 45, 233

Vauclair, S., Dolez, N., \& Gough, D. 1991, A\&A, 252, 618

Wade, G. A., Bohlender, D. A., Brown, D. N., et al. 1997, A\&A, 320, 172 